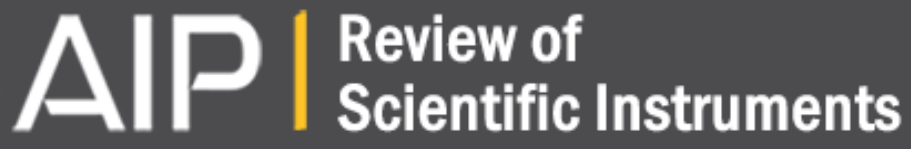

\section{Fast switching of magnetic fields in a magneto-optic trap}

C. J. Dedman, K. G. H. Baldwin, and M. Colla

Citation: Review of Scientific Instruments 72, 4055 (2001); doi: 10.1063/1.1408935

View online: http://dx.doi.org/10.1063/1.1408935

View Table of Contents: http://scitation.aip.org/content/aip/journal/rsi/72/11?ver=pdfcov

Published by the AIP Publishing

\section{Articles you may be interested in}

An efficient magneto-optical trap of metastable krypton atoms

Rev. Sci. Instrum. 81, 123106 (2010); 10.1063/1.3520133

Transferring cold atoms in double magneto-optical trap by a continuous-wave transfer laser beam with large red detuning

Rev. Sci. Instrum. 79, 123116 (2008); 10.1063/1.3058607

High efficiency magneto-optical trap for unstable isotopes

Rev. Sci. Instrum. 74, 4342 (2003); 10.1063/1.1606093

Fast imaging polarimeter for magneto-optical investigations

Rev. Sci. Instrum. 72, 2661 (2001); 10.1063/1.1368855

Fast loading of a magneto-optical trap from a pulsed thermal source

J. Appl. Phys. 84, 6499 (1998); 10.1063/1.369018

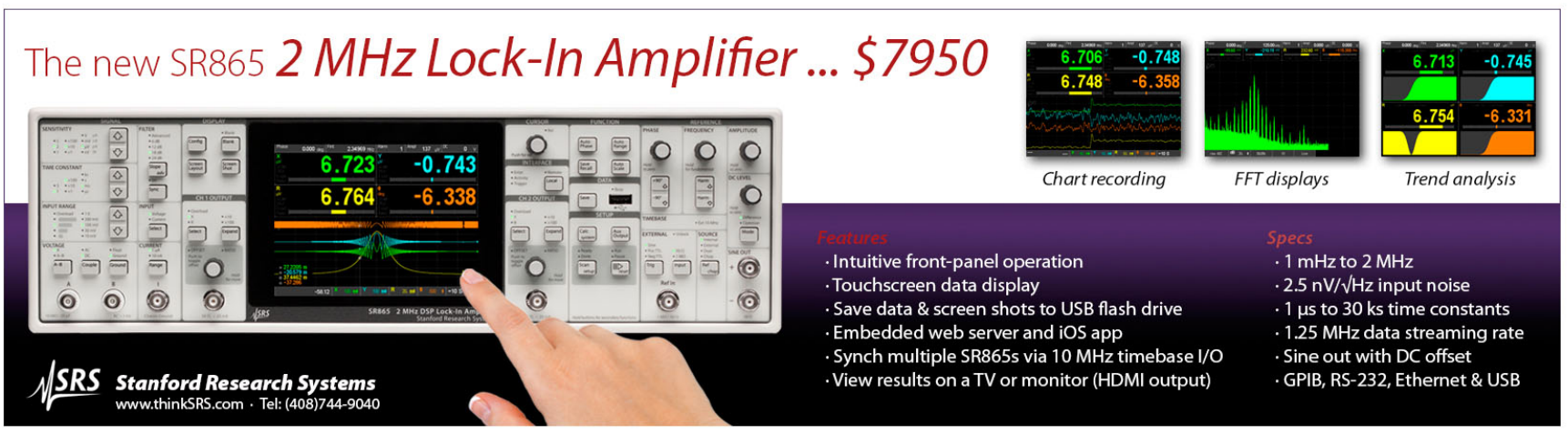




\title{
Fast switching of magnetic fields in a magneto-optic trap
}

\author{
C. J. Dedman, K. G. H. Baldwin,, and M. Colla \\ Research School of Physical Sciences and Engineering, Australian National University, Canberra ACT 0200, \\ Australia
}

(Received 18 April 2001; accepted for publication 13 August 2001)

\begin{abstract}
Magneto-optic traps which employ current windings to generate pulsed magnetic fields require rapid switch-off times for many applications. Practical difficulties in attaining rapid switch-off of the magnetic field, including the generation of induced currents, are addressed. Several methods for minimizing the switch-off time are presented which do not require complex feedback mechanisms involving direct measurement of the magnetic field. (C) 2001 American Institute of Physics.
\end{abstract}

[DOI: $10.1063 / 1.1408935]$

\section{INTRODUCTION}

The magneto-optic trap (MOT) used for the confinement of laser-cooled atoms in a high vacuum environment has become the workhorse for many experiments in atomic, optical, and other fields of fundamental physics. The MOT employs a quadrupole magnetic field (in combination with counterpropagating, red-detuned laser beams) to create a restoring force that confines the atoms at the zero magnetic field point in the trap center. ${ }^{1}$ The quadrupole magnetic field is usually generated by two air-cored, current-carrying coils producing opposing fields to create a constant radial magnetic field gradient originating from the magnetic field zero.

The MOT is just one of many experiments that employ electrical windings to rapidly switch or modulate magnetic fields. Examples include particle storage rings, ${ }^{2}$ electron beam lithography, ${ }^{3}$ and magnetic resonance imaging (MRI) scanners. ${ }^{4}$ A related application is the active magnetic shielding of electron microscopes ${ }^{5}$ and biomagnetic experiments, ${ }^{6}$ where electrical coils are used to dynamically cancel external magnetic fields at frequencies up to $\sim 1 \mathrm{kHz}$.

In the case of the MOT, rapid magnetic field switching is often required to enable other optical fields to be turned on quickly without residual magnetic-field shifting of the atomic energy levels (for example, in polarization gradient cooling). In our particular application, we are interested in obtaining electron-scattering collision cross sections ${ }^{7}$ that are determined by measuring the loss rate of trapped atoms, caused by the impact of electrons in the energy range $10-100 \mathrm{eV}$. The electrons are generated by an electron gun inside the trap vacuum chamber and their trajectories can be perturbed severely by the presence of stray magnetic fields. It is, therefore, crucial to our experiment that the magnetic field is reduced rapidly to zero to enable the electron scattering measurement to take place before the trapped atoms expand ballistically from the trap region on millisecond time scales.

Reducing the winding current itself to zero rapidly is relatively straightforward and is usually limited only by the maximum coil voltage that can be tolerated as the coil current decays. However, in many circumstances the magnetic

${ }^{a)}$ Electronic mail: kenneth.baldwin@anu.edu.au field may decrease much slower than the winding current if the winding is close to electrically conducting objects such as vacuum chambers, flanges, support structures, etc. Such objects effectively form a "shorted turn" secondary winding, and eddy currents induced in this "winding" will decay at a relatively slow rate dictated by the inductance and resistance of the conducting objects.

Similar problems arising from induced eddy currents are encountered in the other applications mentioned above. In the case of high speed magnetic deflection coils for electron beam lithography, eddy current effects can be minimized through careful use of materials, ${ }^{3}$ or by shielding the deflection magnet within a ferrite cylinder. ${ }^{8}$ Neither approach is practical for the MOT which utilizes a complex metal vacuum chamber that requires a high degree of optical access for the laser beams.

MRI machines employ "gradient coils" that typically switch in several hundreds of microseconds, and eddy currents can again slow the gradient response and distort the fields. Eddy-current compensation circuits have been employed where the coil driving current is modified so as to cancel the eddy-current field contribution. ${ }^{4}$ Similar open- and closed-loop compensation methods have been applied to the storage-ring beam-correction magnets ${ }^{2}$ where eddy currents in the half-inch thick aluminum vacuum chamber caused serious amplitude and phase degradation of the beam-positioncorrecting magnetic field, even at the low operating frequency of around $10 \mathrm{~Hz}$.

In this article we describe a simple eddy-current compensation system that does not rely on feedback for its operation. This is important for MOT applications, since it is virtually impossible to monitor the magnetic field near the trapped atoms while the experiment is in operation.

\section{EXPERIMENTAL DESCRIPTION}

We employ metastable helium atoms in the long-lived $2{ }^{3} S_{1}$ state as the target for our electron scattering measurements. The excited-state atoms are generated in a liquid nitrogen-cooled discharge source ${ }^{9}$ and are loaded into the 


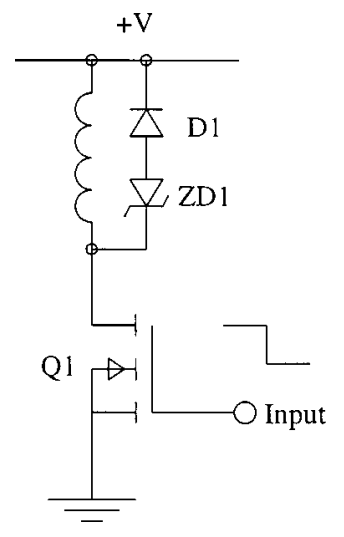

FIG. 1. Schematic diagram of the circuit for rapidly switching the coil current.

MOT by a "bright" atomic beam line which collimates, slows, and compresses the metastable helium beam prior to loading. ${ }^{10}$

The MOT comprizes a multiport, stainless steel vacuum chamber with two reentrant windows around which the magnetic field coils are placed. The water-cooled brass former on which the coils are wound is cut so that a secondary winding is not created, thereby eliminating one source of eddy currents. However, the same clearly cannot be done for the stainless steel vacuum chamber.

Each magnetic field coil consists of $\sim 100$ turns of 1.6 $\mathrm{mm}$ diameter enameled copper wire. Winding inductance with both windings connected in series is $3.75 \mathrm{mH}$, the total resistance is $0.85 \Omega$, and the normal winding current is around $4 \mathrm{~A}$ for standard MOT operating conditions. Each loading and experimental cycle operates at a rate of $\sim 10 \mathrm{~Hz}$. We require that the decay time for the magnetic field to reach $1 \%$ of its maximum value be much less than a millisecond in order to turn on the electron gun before the trap density has decayed significantly.

Every current winding forms an inductor, for which the following relationships between the back $\operatorname{emf}(\epsilon)$, the inductance $(L)$, the current $(I)$, the stored inductive energy $(E)$, and the resistance $(R)$ apply:

$$
\begin{aligned}
& \epsilon=-L \frac{d I}{d t}, \\
& e=\frac{1}{2} L I^{2}, \\
& I=I_{0} \exp ^{[-t /(L / R)]} .
\end{aligned}
$$

To maximize $d I / d t$ the inductor must be permitted to generate a large back emf and a trivially simple circuit for this purpose is shown in Fig. 1. Zener diodes are available to at least $200 \mathrm{~V}$, and higher zener voltage and power dissipation is easily obtained by using several in series. Note that $Q 1$ must be selected to withstand the sum of the rail voltage and the winding back emf.

In some cases the zener diode can be omitted altogether and the metal-oxide-semiconductor field effect transistor (MOSFET) avalanche breakdown voltage used to clamp the winding back emf. For example, we used a $100 \mathrm{~V}$ rated power MOSFET type IRF3710, with a published repetitive

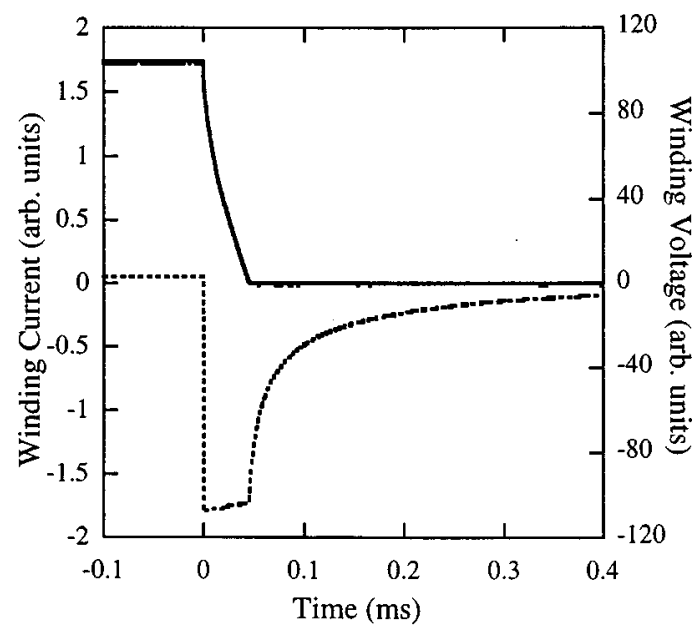

FIG. 2. Winding current and voltage wave forms for the circuit used in Fig. 1. Solid line: winding current. Dashed line: winding voltage.

avalanche dissipation of $20 \mathrm{~mJ}$, and single-shot rating of 530 $\mathrm{mJ}$ at $28 \mathrm{~A}$. From Eq. (1) our stored inductive energy is 30 $\mathrm{mJ}$ at $4 \mathrm{~A}$, which should be within the MOSFET ratings, given our low repetition rate of $\sim 10 \mathrm{~Hz}$.

Figure 2 shows the measured winding current and voltage for the circuit of Fig. 1, with the current decreasing linearly to zero in $50 \mu \mathrm{s}$. Faster decay times could be achieved by selecting a higher-voltage MOSFET switch and zener diode. Zener diode ZD1 can be replaced by a resistor, in which case the coil current decreases exponentially to zero with a decay time constant $L / R$ as in Eq. (3). However, the linear current decay obtained using a zener diode reaches zero much faster without the need to wait for several time constants, and is therefore preferable.

Ideally, we would directly measure magnetic field strength decay time with a fast responding Hall effect sensor or similar device placed within our vacuum chamber, but this is impractical in the case of the MOT. Instead, a 40-mmdiameter pick-up coil consisting of a dozen turns was placed outside the chamber and inside the former of one of the field coils. The induced voltage is proportional to the rate-ofchange of magnetic flux $d \varphi / d t$, but a plot of flux versus time can be obtained by integration. In practice we can also measure the effect of the decaying magnetic field on the temporal shape of the current pulse from the electron gun, which is the ultimate test of the performance of the magnetic-field compensation system.

Figure 3 shows the pick-up coil signal when the magnet winding current is switched to zero in $50 \mu$ s using the circuit of Fig. 1. Analysis of Fig. 3 shows that the flux falls to zero only after $1.5 \mathrm{~ms}$, and this was confirmed in our experiment where the electron beam current was seriously perturbed by the magnetic field up to $1.5 \mathrm{~ms}$ after the coil current was switched off. This is a very long decay time for the magnetic field, given that the magnet current was reduced to zero within $50 \mu \mathrm{s}$. The long decay is the result of conducting materials in the vacuum chamber and other nearby objects, effectively forming a shorted single-turn secondary transformer winding. The resulting induced eddy current creates a 


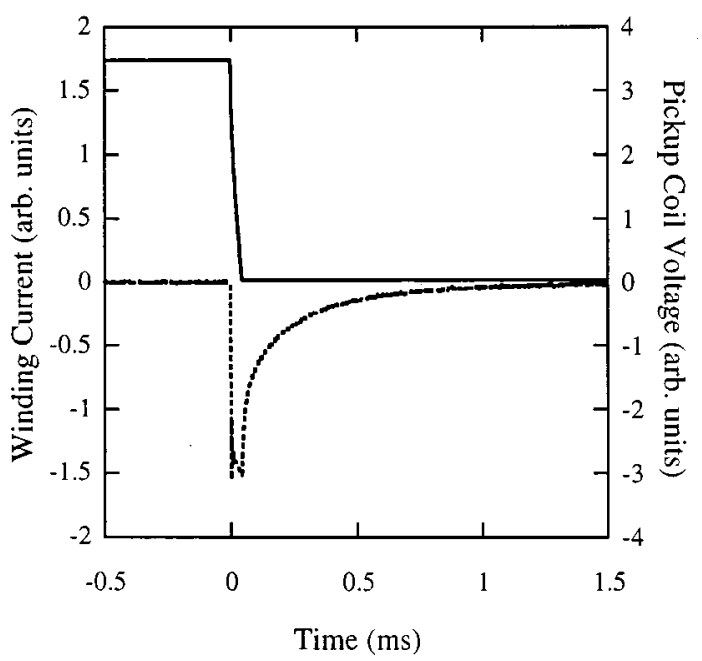

FIG. 3. Winding current and pickup coil voltage wave forms for the circuit shown in Fig. 1. Solid line: winding current. Dotted line: pickup coil voltage. Note that the magnetic flux (indicated by the pickup coil voltage) decays much more slowly than the magnetic coil current.

magnetic flux, which decays exponentially with time, as in Eq. (3).

Note that the slowly decaying magnetic field also induces a voltage in the main magnet windings as shown in Fig. 2, where the winding voltage decays slowly to zero even though the winding current is brought to zero in $50 \mu \mathrm{s}$. However, the pick-up coil voltage is a better diagnostic of magnetic flux at the trap center as it can be placed closer to the trap region, where the flux and pick-up voltage will be somewhat different.

As a first step towards reducing the decay time, every effort should thus be made to minimize conducting structures in the vicinity of the magnet coil, particularly rings or cylinders which form a complete shorted turn. For example, coil formers should be nonmetallic, or should be completely cut at one point on the circumference as in our experiment. We tested a magnet coil similar to that used in the experiment in an area free of metallic objects, and the flux measured by a pick-up coil fell to zero within $30 \mu$ s, in exact sympathy with the coil current.

\section{EDDY-CURRENT COMPENSATION RESULTS}

In our atom-trapping apparatus it should be possible to null the slowly decaying magnetic field by driving the coil

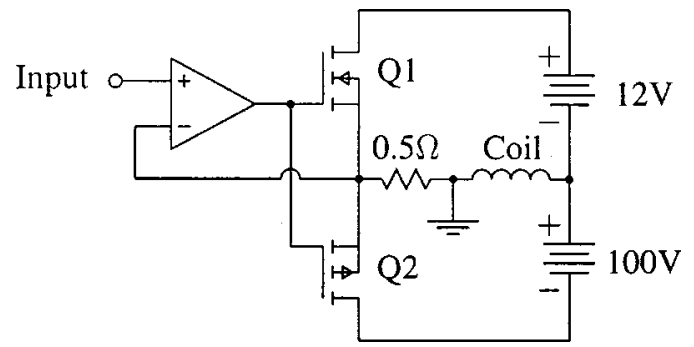

FIG. 4. Simplified schematic diagram of the bipolar current amplifier for providing a current compensation wave form to rapidly decrease the magnetic field to zero.
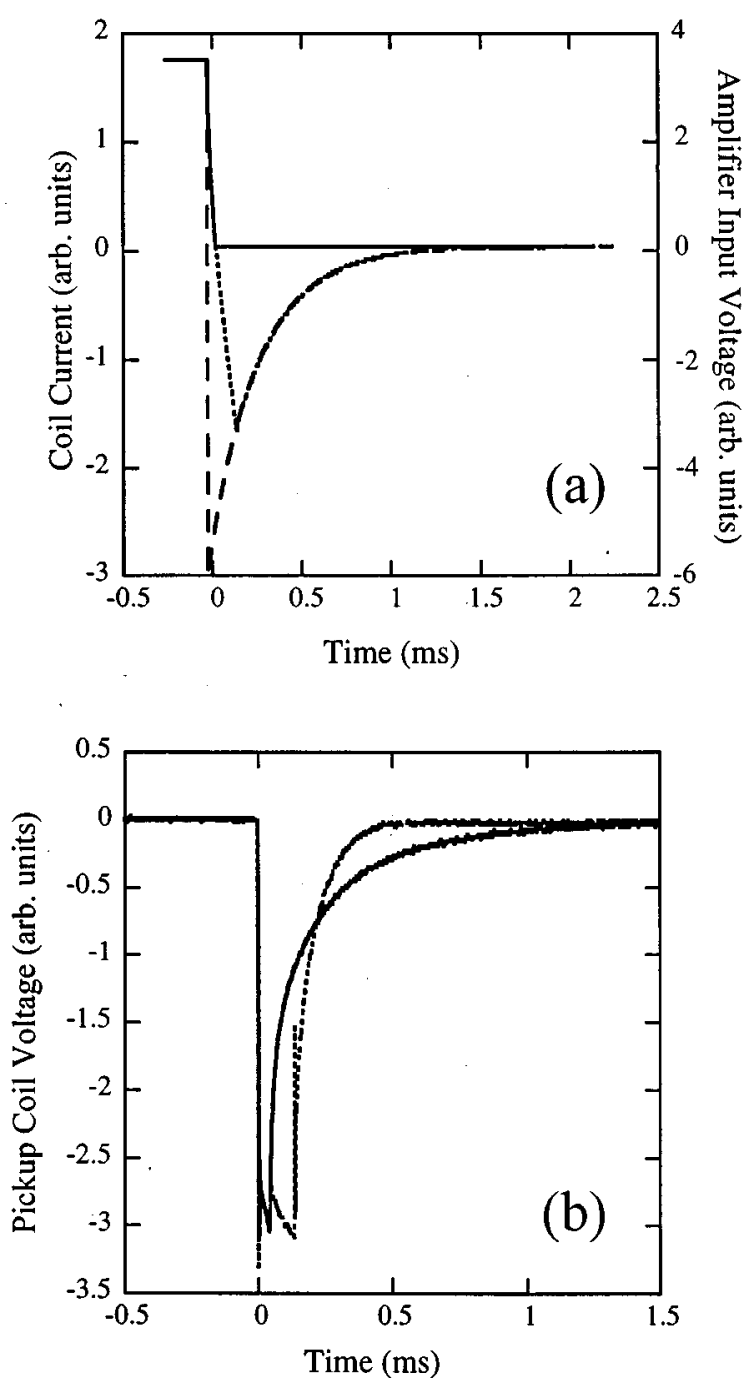

FIG. 5. Wave forms for the current compensation circuit shown in Fig. 4. (a) Solid line: winding current without compensation. Dotted line: current with compensation. Dashed line: amplifier input voltage. (b) Solid line: pickup coil voltage without compensation. Dotted line: voltage with compensation. The decay time of the magnetic field is greatly reduced by the addition of the reverse compensating current.

with an opposing current such that the net magnetic field produced is zero. To test this proposition, a fast response bipolar current amplifier was constructed, a simplified schematic circuit for which is shown in Fig. 4. The coil current faithfully follows the amplifier input voltage without "ringing" or overshoot, subject to $d I / d t$ limitations imposed by the amplifier rail voltages. In our case $d I / d t$ is required to be large only in the negative direction, so amplifier dissipation is greatly reduced by using only $12 \mathrm{~V}$ for the positive supply. Zener diodes are not required to clamp the coil back emf, the decaying coil current being absorbed by the power-supply rail, via the FET body diode. Ideally, a magnetic sensor could provide a feedback signal to the amplifier such that the measured magnetic field is rapidly brought to, and maintained at, zero.

As mentioned previously, it is not possible to place a sensor within our atom trap, so a different approach was used. The required compensating current wave form is, to a very good approximation, exponential in nature. Therefore a 
simple $R C$ circuit was used to generate a negative exponential decay voltage at the amplifier input, with the wave form determined by just two parameters: the amplitude and the decay time constant. In the present experiment, these parameters were simply adjusted in situ using potentiometers. (A digital-to-analog converter driven from a software program could equally well be used to generate the compensating wave form.) In practice, it was a simple matter to adjust the potentiometers to bring the magnetic field to zero in the shortest time, either by observing the output from our pick-up coil, or by noting the perturbation of the electron beam.

Figure 5 shows the amplifier input voltage, magnet coil current, and the pick-up coil voltage with and without the reverse correcting current applied. The pick-up coil voltage shows that the magnetic field decays to zero substantially faster when the reverse compensating current is applied. In our atom trapping experiment, the electron beam could be switched on around $350 \mu \mathrm{s}$ after the magnet was switched off, compared to $1400 \mu$ s previously, an improvement of a factor of 4. By generating a more complex compensating current wave form and by increasing the negative rail voltage in the circuit shown in Fig. 4, the decay time could, in principle, be reduced even further. However, the present reduction was sufficient for the purposes of the experiments considered here.

\section{DISCUSSION}

Very simple circuits can rapidly switch coil winding currents to zero, but the magnetic field may take much longer to decay due to eddy currents induced in conducting objects near the winding. In such cases, the magnetic field decay time can be reduced substantially by driving the magnet winding with a suitable compensating current wave form. Here we have demonstrated that effective compensation in a MOT can be achieved using a simple two-parameter waveform characterization, without requiring a more complex (and, in the case of a MOT, impractical) system involving measurement of the magnetic field and subsequent feedback to the current wave-form generator. In our MOT experiment, the magnetic field decay time was readily reduced by a factor of 4 (to around $350 \mu \mathrm{s}$ ) compared to that achieved by simply turning off the magnet winding current. Further improvement in the decay time may be achieved by more precise tailoring of the compensating current wave form.

\section{ACKNOWLEDGMENTS}

The authors would like to thank John Close for helpful discussions and, along with Brenton Lewis, for careful reading of the manuscript.

${ }^{1}$ E. Raab, M. Prentiss, A. Cable, S. Chu, and D. E. Pritchard, Phys. Rev. Lett. 59, 2631 (1987).

${ }^{2}$ Y. Chung, Argonne National Laboratory LS notes 148 (1990) and 209 (1992), www.aps.anl.gov/techpub/lsnotes/ls148/ls148.html.

${ }^{3}$ H. T. Pearce-Percy, D. F. Spicer, M. Abbot, C. Winborn, and G. L. Varnell, J. Vac. Sci. Technol. 16, 1794 (1980).

${ }^{4}$ D. J. Jensen, W. W. Brey, J. L. Delayre, and P. A. Narayana, Med. Phys. 14, 859 (1987).

${ }^{5}$ S. D. Golladay, J. Vac. Sci. Technol. B 6, 2070 (1988),

${ }^{6}$ D. Platzek, H. Nowak, F. Giessler, J. Rother, and M. Eiselt, Rev. Sci. Instrum. 70, 2465 (1987).

${ }^{7}$ M. Jacka, M. D. Hoogerland, W. Lu, D. Milic, K. G. H. Baldwin, K. Bartschat, and S. J. Buckman, J. Phys. B 29, L825 (1996).

${ }^{8}$ L. H. Lin and H. L. Beauchamp, J. Vac. Sci. Technol. 10, 987 (1980).

${ }^{9}$ W. Lu, M. D. Hoogerland, D. Milic, K. G. H. Baldwin, and S. J. Buckman, Rev. Sci. Instrum. 72, 2558 (2001).

${ }^{10}$ M. D. Hoogerland, D. Milic, W. Lu, H.-A. Bachor, K. G. H. Baldwin, and S. J. Buckman, Aust. J. Phys. 49, 567 (1996). 